\title{
Optimization of the propagation model choice by measuring field and artificial intelligence
}

\section{Alberto Leonardo Penteado Botelho \\ Cristiano Akamine}




\title{
Optimization of the propagation model choice by measuring field and artificial intelligence
}

\author{
Alberto Leonardo Penteado Botelho, Cristiano Akamine
}

\begin{abstract}
The propagation model to be chosen in the design of a digital terrestrial broadcast station is a tipping point for predicting the coverage area. There are several models, with specific characteristics that may be better than others in certain situations. This paper presents a study of the choice of propagation model, through the use of artificial intelligence (AI). A brief review of the most widely used propagation models in the literature, field measurements and simulations by the Progira coverage prediction software, which operates on the ArcGIS geoprocessing platform are presented. Using the propagation model criterion that presents the smallest error between the field measurement and the software simulation, an AI method of classification learning was developed. The objective of this method can choose, with the smallest error, the best propagation model in the entire study area, not restricted to the Sites measured in the field.
\end{abstract}

Index Terms - Classification Learner, Artificial Intelligence, Field Measurement, Propagation Model, Single Frequency Network.

\section{INTRODUCTION}

$\mathrm{T}$ he Technical project is essential for the deployment of terrestrial television broadcasting stations, whether analog or digital, as it allows a broadcaster to predict the coverage area. Coverage area is the region where the signal level transmitted by the television station allows decoding on TV sets.

As ISDB-TB (Integrated Services Digital Broadcasting Terrestrial version B) digital television technology allows operation with SFN (Single Frequency Network) stations, it is possible that coverage failures can be corrected by deploying auxiliary transmitters at the same frequency to improve the coverage area. In this way, the prediction of the coverage area and the field measurement become even more critical and dependent.

Most complex coverage area prediction softwares use the point-by-point method, which requires sophisticated algorithms.

Regardless of the software used, the tipping point is the choice of the propagation model that best suits the characteristics of the study region.

There are several propagation prediction models found in the technical literature, such as Okumura Hata, Longley Rice, Ordinance 53 of Ministério das Comunicações, Recommendation ITU-R 1546-1, but they are not always sufficient to characterize reception conditions in isolation [1]
The difficulty in finding the best propagation model evidences that there is no an ideal propagation model for each case and deviations are common from what was simulated and measured in the field. Field measurement becomes a way of adjusting the coverage area of the transmitter.

In order to improve the relationship between coverage area prediction and field measurement, this study is not intended to create a new propagation model, but to establish a method of choosing the best propagation model for a specific area and, if necessary, to establish a model of hybrid propagation for a given region.

Thus, a comparison was made between field measurements and software simulations, in order to establish criteria to predict the best model of propagation of non-field-measured sites.

The Progira coverage area prediction software was used and provided by LM Telecom [2] and the field measurement in the metropolitan area of Rio de Janeiro has been made available by Record Rio (Televisão Record do Rio de Janeiro Ltda.).

On the research, the most important criteria that determine which model of propagation was appropriate for each measured site have been approached and, therefore, present more reliable criteria for choosing the propagation model in the regions not measured in the field.

Point-by-point simulation software presents its predictions on a large number of points located in the study region, so a suitable computational tool must be created to process this information.

Each predicted site has a field strength value calculated by the propagation model chosen. It is possible to set a specific color for field strength with values equal to or above the stipulated and to form a limited noise coverage.

The limited noise coverage shows the regions with field strength above the stipulated, which represents good coverage and also demonstrates regions with field strength below the stipulated, which represents poor coverage and that should be addressed to improve the coverage area.

A program has been developed using AI to make the most appropriate propagation model decision making for each chosen measurement site.

The programming language chosen to implement the AI program was Matlab, which has Neural Network (NN) applications developed to support propagation model choice decisions. Matlab has been chosen because it has a set of NN and AI toolboxes. Matlab uses Jit Accelerator technology, 
which substantially increases the execution performance of applications, compared to other programming languages such as $\mathrm{C} / \mathrm{C}++$, Foltran or Visual Basic [3].

This study is aimed at optimizing the coverage area prediction method so that broadcasters can install their systems with a high guarantee that the coverage area is consistent with the plan, minimizing new measures for correction of cover not provided in the software simulations.

This article is divided into seven sections in addition to this introductory section. In Section II, a brief description of the SFN operation, the importance of the coverage simulations and the complexity of choosing the best propagation model will be presented. In Section III, the methodology of the prediction simulations of the coverage area, the propagation models used and the equations for the database to be used by the IA will be presented. In Section IV the methodology of field measurements will be demonstrated. In Section V, AI techniques that can aid in decision making and formulate aspects of human intelligence that can be reproduced by machines will also be presented. In Section VI, the results of the application of AI in the choice of propagation model will be demonstrated. Finally, Section VII will present the main conclusions and final considerations of the work done.

\section{SFN OPERATION}

The set of essential functionalities required in digital television reception devices is regulated by the ABNT (Brazilian Association of Technical Standards). For Digital Terrestrial Television receivers, the ABNT standards [4] recommends that the minimum level of input to the receiver must be greater than or equal to $-77 \mathrm{dBm}$ for operation in mode 3, 1/8 guard interval, no time interleaving, 64 QAM modulation and $3 / 4$ code rate.

Brazilian legislation defines SFN as a set formed by the generating station and auxiliary or retransmitters that operate on the same channel and transmit precisely the same content simultaneously. The viable channel for the retransmitter stations must be identified in the PBTVD (Basic Digital Television Plan) as reusable and its technical characteristics in it. Channel reuse is the reuse of the same channel used to provide the service granted [5].

In order to allow operation according to the distance between the stations of a SFN and ensure proper reception channel variations as a result of the Doppler effect of the mobile reception signal, one can select among three spacing options of OFDM carriers offered by the Brazilian system. These three must be identified as system modes.

In the case of Brazil, the frequency spacing shall be approximately $4 \mathrm{kHz}, 2 \mathrm{kHz}$ or $1 \mathrm{kHz}$, respectively for modes 1,2 and 3 . The number of carriers varies depending on the mode, but the useful bit rate of each mode must be precisely the same in all modes [6]. The different mode settings, modulation method, guard interval and convolutional code significantly change the coverage area, data rate and minimum distance between the carriers that allows synchronization in SFN.

The SFN operation can significantly improve spectrum efficiency, service quality and reduce power emission compared to single-transmitter networks. SFN uses several transmitters that operate on the same RF channel, transmitting the same programming, which can adequately cover a large geographic area [7].

The SFN operation is very advantageous, but it entails the need for a much more rigorous planning. SFN offers many potential advantages, the greatest of which is the reduction of interference between different TV stations and greater flexibility of coverage, but this system should be planned with great care, especially in marginal performance [8].

If a single electromagnetic wave of only one transmitter arrives at a receiver without any channel distortion, then it is easy for the receiver circuit to estimate the phase and quadrature components of the symbols carried by each OFDM frequency carrier. When comparing performance in the SFN scenario, the $\mathrm{C} / \mathrm{N}$ (Carrier to Noise Ratio) required for acceptable reception is greater than in the single arrival signal scenario where there is a loss of reception margin [9].

Simulations, based on a case study, show that gap filler stations used in metropolitan urban regions are useful and necessary tools for improving digital TV coverage, covering some regions that may be obstructed by buildings, vegetation, relief or any other kind of obstacle [10]. This statement evidences the trend of installing SFN systems.

The simulations are aimed at predicting the coverage area. The coverage area is determined by the field strength value of the received signal, which must be adequate for the conditions of reception of the studied locality considering the environmental, urban and topographical characteristics [1].

When making reliable predictions of coverage, the number of measurements to be performed in the scenario of interest is considerably reduced, also implying a decrease in time and cost of the project [11].

Uncertainties in predicting coverage can also make engineers opt for more conservative transmission systems to ensure the lowest coverage, with higher radiated power. The higher the power, more areas of intersection of the network, increasing the loss of reception margin.

\section{SOFTWARE SIMULATION}

With the values of field strength measured in each of the 44 Sites, a calculation has been made to predict the coverage area. In the calculations, Record Rio's SFN transmissions have been considered under the same installation conditions at the time of measurements and under the same field measurement reception conditions.

Figure 1 shows a map with an example of an area with the transmitting station, located in Rio de Janeiro, at Morro do Sumaré, and 4 of the 44 sites where field measurements have been taken.

The software used to predict the coverage area was Progira, [12] which operates on the ArcGIS platform, which creates and shares maps, scenes, applications, layers, analytics and data [13].

Coverage prediction software operates with propagation model, which is a method based on interpolation / extrapolation of empirically derived field strength curves such as distance functions, antenna height, terrain clearance, and link obstructions [14].

There is no single criterion for deciding the best model based on the comparison of experimental data. The most frequently used statistical measures are the mean and standard deviation of errors. There are other issues related to 
the behavior and general nature of a model that can be decisive [15].

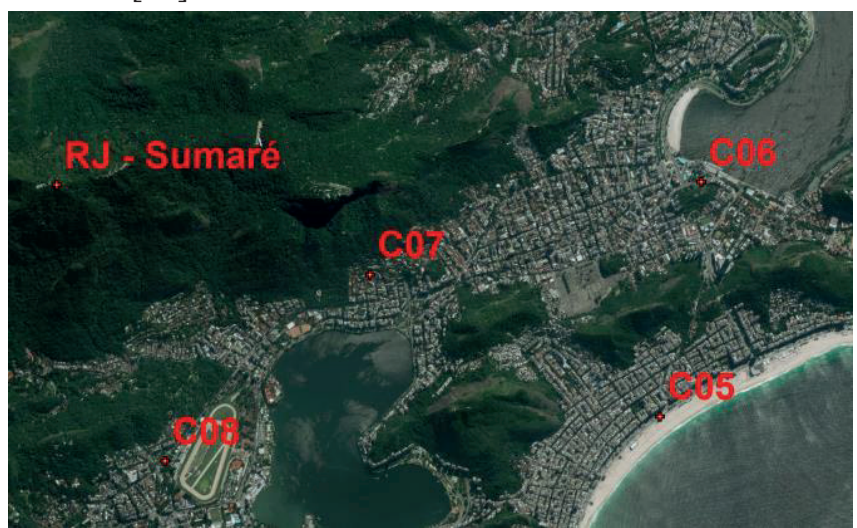

Figure 1: Map area with some transmitter and field measurement sites

Progira software currently offers ten propagation models, which are CRC-Predict, ITUR P.1546-5, ITUR P.1546-4, ITUR GE06, ITUR P.370-7, ITUR P.526-13, ITUR P. 52611, ITUR P.1812-3, ITUR P.11812-2, Deygout-Assis, Longley-Rice, Okumura-Hata and Free Space. Each propagation model has some kind of option, such as climate, types of obstacles or types of population density, that increased the number of models of propagation to 37 .

1. CRC-Predict has the options of Continental climate, Great Lakes, Maritime on Earth and Maritime on the Ocean.

2. Deygout-Assis has the options of Rounded Terrain, Main Round and Knife Edge.

3. Free Space has no options;

4. ITUR P.370-7 has options for Rural, Suburban and Urban geographic regions.

5. ITUR P.526-11 has options for Round Terrain and General Method.

6. ITUR P.1546-5 has options for rural / open, suburban, urban and dense urban geographic regions.

7. ITUR P.1812-3 has geographic options with software database, Forest, Suburban, Urban and Denso Urban.

8. ITUR GE06 has geographic options of region Rural/ Open, Suburban, Urban and Urban Denso.

9. Longley-Rice has geographic options for Subtropical Continental Climate Options, Equatorial Continental, Desert, Equatorial, Tropical Seas, Temperate Seas on Earth and Temperate Seas on the Ocean.

10. Okumura-Hata has geographical option for Open, Quasi-Open, Suburban and Urban.

Thus, the software calculated the field strength of 11 stations, repeated for each of the 37 propagation models, totaling 407 limited noise coverages.

Anatel has a recommendation for survey accuracy for the average terrain level. The average terrain level for each radial shall be raised in at least 12 directions from the antenna site, taking into account the stretches between $3 \mathrm{~km}$ and $15 \mathrm{~km}$. The radial ones must be traced with an angular spacing of $30^{\circ}$ each other, including the direction of the True North [16].

Considering the current high computational capacity, the accuracy of each point was increased to $50 \mathrm{~m}$ spacing in steps of $1^{\circ}$.

The propagation models calculate the field strength by the effective distance and height between the transmission and the average terrain level.

As the simulation software presents values in $\mathrm{dB} \mu \mathrm{V} / \mathrm{m}$, and the field measurement presents values in $\mathrm{dBm}$, it was necessary to convert the field measurement values to $\mathrm{dB} \mu \mathrm{V} / \mathrm{m}$, since it presents smaller values for conversion. The equation of the conversion is demonstrated in Equations (1, 2, and 3).

$E(d B \mu V)=20 \times \log 10[E(V)]$

$K(d B)=-29,77-14+2+(\log 10 \times 623)$

$E(d B \mu V / m)=E(d B \mu V)+K(d B)$

Where:

$P(m W)=10^{P(d B m) \div 10}$

$P(W)=P(m W)^{-3}$

$Z(\Omega)=75$

$U(V)=P(W) \times Z(\Omega)$

$E(V)=\sqrt{U(V)}$

$E(\mu V)=E(V) \div 10^{-6}$

With the field strength values measured at each of the 44 sites, a prediction calculation of the coverage area was performed. In the calculations, Record Rio's SFN transmissions have been considered under the same installation conditions at the time of measurements and under the same field measurement reception conditions.

Progira has the option to analyze the characteristics of the link path, which can be either visual or data. Figure 2 shows an example of the visual link transmitted from Rio de Janeiro at Morro do Sumaré to Site 06.

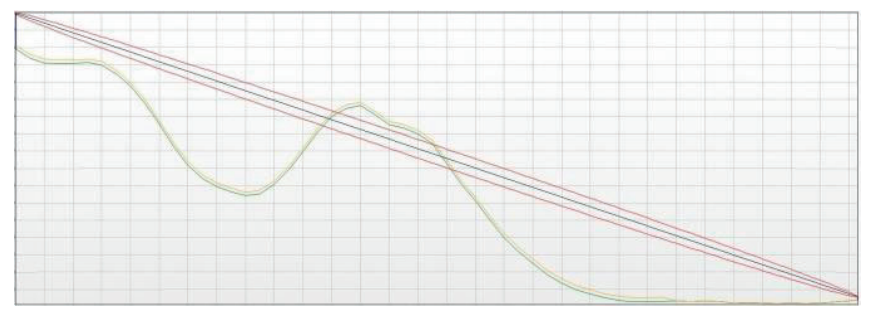

Figure 2: Link between transmission and reception on Site 06

The red line indicates the Fresnel curve, between the transmission at the highest point and the reception at the lowest point. The green line indicates the height of the terrain relative to sea level. The yellow line indicates the clutter, with information of the natural or artificial obstacles.

It is observed that the link transmitted from Rio de Janeiro at Morro do Sumaré to Site 06 is obstructed and has no direct vision.

Table 1 shows an example of the data link of the first kilometer transmitted from Rio de Janeiro at Morro do Sumaré to Site 06.

Table I shows for every 100 meters, the elevation of the land about sea level, the clutter code, the height of the clutter and the description of the clutter.

The accuracy of steps of 100 meters was defined to maintain the methodological criterion already adopted.

The relief database generated for each link displays different amounts of values. A $5 \mathrm{~km}$ link will have 50 values, while a $10 \mathrm{~km}$ link will have 100 values, but for AI to be efficient, all links must have the same amount of values.

Progira can not calculate the link by number of sites, so FFT (Fast Fourier Transform) approximation interpolation was performed so that all links have the same amount of 
values.

Table I

Data in the first kilometer of the link between the transmission and the reception in the Site 06

\begin{tabular}{|c|c|c|c|c|}
\hline $\begin{array}{c}\text { Dist } \\
(\mathrm{km})\end{array}$ & $\begin{array}{c}\text { Elev } \\
(\mathrm{m})\end{array}$ & $\begin{array}{c}\text { Clutter } \\
\text { code }\end{array}$ & $\begin{array}{c}\text { Clutter } \\
\text { h(m) }\end{array}$ & Clutter description \\
\hline 0.1 & 720.2 & 40 & 10 & $\begin{array}{l}\text { Closed to open }(>15 \%) \text { broadleaved } \\
\text { evergreen or semi-deciduous forest } \\
(>5 \mathrm{~m})\end{array}$ \\
\hline 0.2 & 706.1 & 40 & 10 & $\begin{array}{l}\text { Closed to open }(>15 \%) \text { broadleaved } \\
\text { evergreen or semi-deciduous forest } \\
(>5 \mathrm{~m})\end{array}$ \\
\hline 0.3 & 703.6 & 40 & 10 & $\begin{array}{l}\text { Closed to open }(>15 \%) \text { broadleaved } \\
\text { evergreen or semi-deciduous forest } \\
(>5 \mathrm{~m})\end{array}$ \\
\hline 0.4 & 703.8 & 40 & 10 & $\begin{array}{l}\text { Closed to open }(>15 \%) \text { broadleaved } \\
\text { evergreen or semi-deciduous forest } \\
(>5 \mathrm{~m})\end{array}$ \\
\hline 0.5 & 706.6 & 40 & 10 & $\begin{array}{l}\text { Closed to open }(>15 \%) \text { broadleaved } \\
\text { evergreen or semi-deciduous forest } \\
(>5 \mathrm{~m})\end{array}$ \\
\hline 0.6 & 699.9 & 40 & 10 & $\begin{array}{l}\text { Closed to open }(>15 \%) \text { broadleaved } \\
\text { evergreen or semi-deciduous forest } \\
(>5 \mathrm{~m})\end{array}$ \\
\hline 0.7 & 674.6 & 40 & 10 & $\begin{array}{l}\text { Closed to open }(>15 \%) \text { broadleaved } \\
\text { evergreen or semi-deciduous forest } \\
(>5 \mathrm{~m})\end{array}$ \\
\hline 0.8 & 638.3 & 40 & 10 & $\begin{array}{l}\text { Closed to open }(>15 \%) \text { broadleaved } \\
\text { evergreen or semi-deciduous forest } \\
(>5 \mathrm{~m})\end{array}$ \\
\hline 0.9 & 589.0 & 40 & 10 & $\begin{array}{l}\text { Closed to open }(>15 \%) \text { broadleaved } \\
\text { evergreen or semi-deciduous forest } \\
(>5 \mathrm{~m})\end{array}$ \\
\hline 1.0 & 527.6 & 40 & 10 & $\begin{array}{l}\text { Closed to open }(>15 \%) \text { broadleaved } \\
\text { evergreen or semi-deciduous forest } \\
(>5 \mathrm{~m})\end{array}$ \\
\hline
\end{tabular}

The information in the link database is not sufficient for correct manipulation, but it is possible to develop algorithm with the data.

It has been proposed that each step has the information of the type of terrain, the height between the center of the link to the base of the terrain and the distance of the link.

The information of the type of terrain and the height between the center of the link to the ground base was calculated according to Equations (4, 5, 6 and 7).

$$
\begin{aligned}
& H t=C B T+H c i \\
& A=\arctan (H t+H r-C B R) \div(D E) \\
& H e=(C B R+H r+(\text { passo } \times(\tan (A)) \\
& H e r=H e-C B P
\end{aligned}
$$

Where:

$$
\begin{aligned}
& D E=\text { distance from the link } \\
& C B T=\text { tower base height } \\
& C B R=\text { Height of the base of reception } \\
& H c i=\text { transmission antenna height } \\
& H r=\text { height of the receiving antenna } \\
& H e=\text { Link height }
\end{aligned}
$$

\section{FIELD MEASUREMENT}

Based on the quality assumptions, a field test was carried out for Record Rio, with a primary transmission coming from the City of Rio de Janeiro. Rio de Janeiro has been chosen because it is the city that has the most significant inconsistency between measured and simulated values in software. One possible cause of this inconsistency is related to the effective relief in the State, very varied, with high escarpments, seas of hills, hills and valleys, several rocks, besides an extensive area of plateau that occurs in all the west of the territory.

Record Rio operates in the City of Rio de Janeiro at Morro do Sumaré in SFN with two retransmitters, located in the City of Rio de Janeiro, Morro do Mendanha and Pena, duly registered in the Mosaic System of the National Telecommunications Agency - Anatel [17].

Record Rio's SFN network comprises 11 auxiliary retransmitters, located in the City of Rio de Janeiro and the Cities belonging to its metropolitan ring. The information of the technical characteristics of operation of the SFN network was made available by the broadcaster.

Thus, 44 sites have been strategically distributed in the metropolitan area of Rio de Janeiro, in order to obtain different propagation situations. The measurement was elaborated by the company hired for this service rendering. Record Rio authorized the use of the data for this research.

The 44 Sites have been chosen with the intention of regular distribution in the metropolitan region of Rio de Janeiro, based on the current noise limited coverage and the station facilities information.

\begin{tabular}{|c|c|c|c|c|c|}
\hline \multirow{2}{*}{\multicolumn{2}{|c|}{$\begin{array}{l}\text { DEFAUIT } \\
\text { 29/10/2017 17:27 } \\
\text { ISDB-T PARÁMETROS }\end{array}$}} & \multicolumn{2}{|c|}{ MEDIDAS $3 / 3$} & & \multirow[t]{2}{*}{ TE } \\
\hline & & & & & \\
\hline \multicolumn{2}{|c|}{ Largura de banda: } & $6000 \mathrm{kHz}$ & \multicolumn{2}{|c|}{ Inversão espectral: } & Off \\
\hline \multicolumn{2}{|r|}{ Modo FFT: } & $8 \mathrm{k}$ & \multicolumn{2}{|c|}{ Intervalo de guarda: } & $1 / 8$ \\
\hline \multicolumn{2}{|c|}{ Constelação: } & 16QAM & \multicolumn{2}{|c|}{ Taxa de Código } & $3 / 4$ \\
\hline \multicolumn{2}{|c|}{ Entrelaçamento Te... } & 2 & \multicolumn{2}{|c|}{ Medir Camada: } & $B$ \\
\hline \multicolumn{2}{|c|}{ Camada do TS: } & ALL & \multicolumn{2}{|c|}{ Alarme de Emergênc... } & Off \\
\hline Freq & $623.00 \mathrm{MHz}$ & Potência & $-58.9 \mathrm{dBm}$ & MER & $17.7 \mathrm{~dB}$ \\
\hline \multirow[t]{2}{*}{ Offset } & $143.0 \mathrm{kHz}$ & $\mathrm{C} / \mathrm{N}$ & $>25.9 \mathrm{~dB}$ & CBER & $4.2 \mathrm{E}-03$ \\
\hline & & & & VBER & $<1.0 \mathrm{E}-07$ \\
\hline \multicolumn{6}{|c|}{$\checkmark$ MPEG2 TS trancado: RECORD RIO JANEIRO } \\
\hline \multicolumn{2}{|c|}{ CH 39} & ISDB-T & Ferrame & & r Camada: B \\
\hline
\end{tabular}

The measuring system used an antenna with $14 \mathrm{dBi}$ gain in the central frequency of $623.143 \mathrm{MHz}$, corresponding to the TV Channel 39, with height of 10 meters and cable attenuation of $2 \mathrm{~dB}$.

As Record Rio has auxiliary retransmitters, the receiving antenna was directed to the signal with greater intensity of field.

Figure 3 shows the technical characteristics measured in Site 06, in layer B with Full Seg programming.

Figure 3 - Technical characteristics of Site 06.

In Figure 3, it is most possible to identify the transmitter station multiplex configuration, such as the 16-QAM modulation, FEC $3 / 4$ and the guard interval of $1 / 8$. It is also possible to identify the quality of the received signal power (Potência), C/N, Code Error Rate (CBER), Code Error Rate post Viterbi (VBER) and Modulation error rate (MER).

Figure 4 shows a constellation at Site 06 , which is the $\mathrm{C} / \mathrm{N}$ parameter and enables viewing if the transmitted symbol is a correct region and can be easily detected by the receiver. 


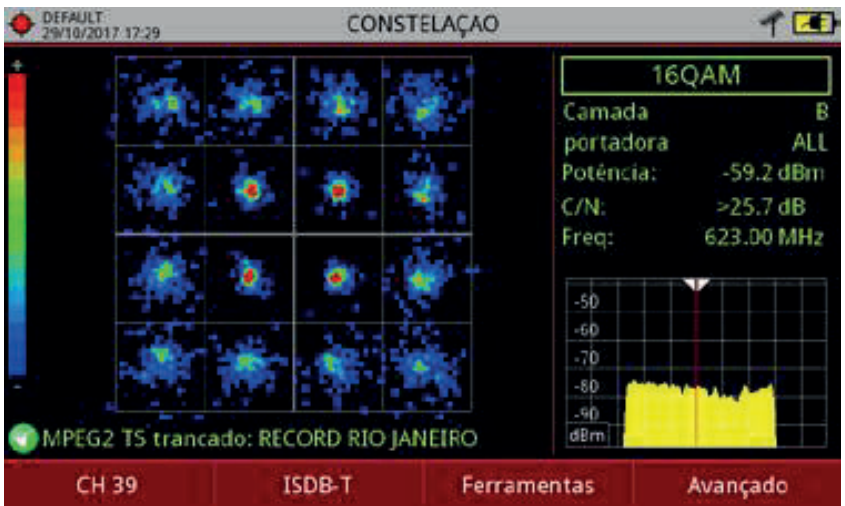

Figure 4 - Constellation of Site 06.

Figure 5 shows the delay profile at Site 06, where it is possible to verify the existence of the primary path with -59.2 $\mathrm{dBm}$, a second path with $-23.6 \mathrm{dBc}$ at $20 \mu \mathrm{s}$ and third path with $-25.1 \mathrm{dBc}$ at $48 \mu \mathrm{s}$.

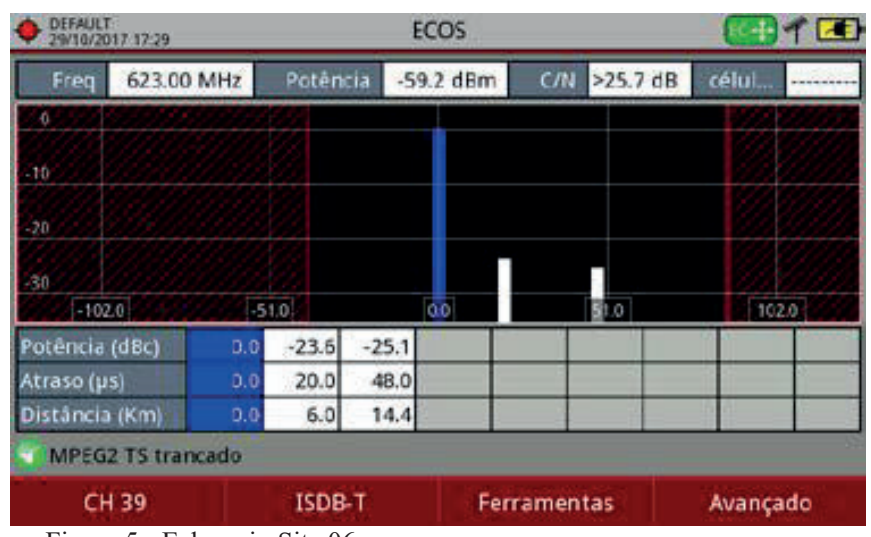

Figure 5 - Echoes in Site 06.

\section{ARTIFICIAL INTELLIGENCE}

Research in AI and, consequently, involving AI-based systems, aims to discover and formulate aspects of human intelligence that can be reproduced by machines. These currently enable building models to perform tasks such as participating in Strategy Games, Recognizing Patterns in Audio and Images, finding proofs of mathematical theorems, and other computational tasks. Currently there are several initiatives involving software and hardware projects with the objective of using AI models to perform complex tasks, such as decision making in electronic negotiations, real-time decisions in dynamic environments [18].

Intelligence is mainly related to the rational action. In the ideal case, an intelligent agent adopts the best possible action in a situation [19]

With AI, you can delegate to the software the decision of choice for each site. An algorithm will be created to predict the best propagation model of each site in the study region.

The system is efficient when we omit information from field measurement and the AI can predict the best propagation model correctly and therefore able to predict all points in the study area.

The literature addresses several AI techniques. Intelligent Systems (IS) can be developed using some fundamental techniques, which can be applied alone or together to aid decision making. The main techniques and methodologies used by IS are: Knowledge Acquisition; Machine Learning;
Neural networks; Fuzzy Logic; Evolutionary Computation; Agents and Multi agents; Data Mining and Texts. Each of these techniques offers a variety of degrees of ability to represent human knowledge. [20]. This statement shows that an in-depth study should be developed to define the appropriate technique to be used in the Study.

This study used the Machine Learning application, since they can model the behavior of the system through the learning process, based on observing performance data over a period. Once properly trained, they can automatically estimate and predict future system behavior and subsequently make admission decisions with high accuracy and speed [21].

The Machine Learning application presents a group neural network tool that allows you to create a digital television signal propagation curve. The network is trained to create a new propagation curve, based on field measurements [22].

Creating a curve with the propagation models may not yield the best result. In order to enable people with muscular disabilities to communicate only with eye movement, the classification learning tool has been applied in the research. The research demonstrates that this training application allows to explore data, select resources, specify validation schemes, train models and evaluate the results [23], so a Machine Learning technique with classification learning application may be more appropriate.

\section{RESULTS}

The field measurement report presented 44 measurements, however, when analyzing the echoes of the field meter, it is observed that in some measurements, it was possible to receive more than one relevant signal. These echoes may be reflected signals or signals from a second transmitter.

It is possible to compare the delay of the echo presented in the field measurement to the delay of the signals of all the transmissions presented in the software simulation. Data considered consistent were also included in the tests.

For these cases, the conversion equations of $\mathrm{dB} \mu \mathrm{V} / \mathrm{m}$ to $\mathrm{dBm}$ needed to be changed in cases, in which the receiving antenna azimuth does not point to the transmitting antenna of the second transmitter. The azimuth loss of the receiving antenna was subtracted from the antenna factor calculation. Thus, the tests considered 52 Sites of measures.

When comparing the values measured in the field to the simulated values by software, it was possible to calculate mean error of each model of propagation, according to Table II.

Table II concludes that the ITUR1812-3 propagation model with clutter for densely urbanized presents the best average error and is the most reliable to be used in the Rio de Janeiro study.

Despite the ITUR1812-3 propagation model, with clutter for densely urbanized presenting the best average error, does not mean that it is the most suitable model in all Sites.

An array was created with the profile data of each link and applied to the classification tool.

The FFT interpolation approximation algorithm can use any number of values. However, it was possible to observe that high values imply a considerable loss of accuracy. It was considered 20 values, to maintain high accuracy without mischaracterizing the terrain information. 
Table II

Average error of each propagation model in the comparison between measured and predicted sites.

\begin{tabular}{|c|c|}
\hline \multicolumn{2}{|l|}{ Average (dB) } \\
\hline ITUR1812-3 - Dense Urban & 9.2 \\
\hline ITUR526-13 - General Method & 10.0 \\
\hline ITUR1812-3 - Forest / Urban & 10.2 \\
\hline $\begin{array}{l}\text { CRC - Continental / Great Lakes / Maritime overland / } \\
\text { Maritime oversea }\end{array}$ & 10.8 \\
\hline ITUR1812-3 - Suburban & 11.0 \\
\hline ITUR1812-3 - Database & 11.0 \\
\hline ITURG06 - Rural / Open / Suburban & 11.2 \\
\hline Deygout Assis - Knife edge & 11.7 \\
\hline Okumura Hata - Quasi Open & 11.9 \\
\hline ITUR370-7 - Rural / Suburban / Urban & 12.1 \\
\hline Deygout Assis - Main Rounded & 12.4 \\
\hline ITUR1546-5 - Rural / Open & 12.5 \\
\hline ITUR1546_5 - Suburban & 12.5 \\
\hline Okumura Hata - Open & 12.8 \\
\hline Longley Rice - Equatorial & 13.1 \\
\hline Longley Rice - Desert & 13.1 \\
\hline Longley Rice - Maritime Temperate oversea & 13.1 \\
\hline Longley Rice - Continental Subtropical & 13.1 \\
\hline Longley Rice - Maritime Temperate overland & 13.1 \\
\hline Longley Rice - Maritime Tropical & 13.1 \\
\hline Longley Rice - Continental Temperate & 13.7 \\
\hline Deygout Assis - Rounded & 13.8 \\
\hline ITUR526-13 - Rounded & 15.8 \\
\hline Okumura Hata - Suburban & 16.1 \\
\hline ITURG06 - Urban & 16.2 \\
\hline Free Space & 16.8 \\
\hline ITUR1546-5 - Urban & 18.1 \\
\hline ITURG06 - Dense Urban & 20.6 \\
\hline Okumura Hata - Urban & 22.1 \\
\hline ITUR1546-5 - Dense Urban & 21.2 \\
\hline
\end{tabular}

Therefore, the matrix has 42 values. The 1 st corresponds to the classification of the propagation model, the 2nd corresponds to the distance of the link, the $3 \mathrm{rd}$ to the $22 \mathrm{nd}$ corresponds to the height between the center of the link to the base of the ground and the $23 \mathrm{rd}$ to the $42 \mathrm{nd}$ correspond to the type information of ground.

The classification learning tool has the overfitting and partial protection options. The non-overfitting protection option, indicated for large amounts of data, was the one that presented the best accuracy.

Each type of learning returns a different value of accuracy according to Table III.

Table III shows that several types of learning present $100 \%$ accuracy, so, they were able to learn the behavior of each link, thus, 21 trained models were created. To test the capacity of the AI, tests were performed on all measured Sites. It was elaborated 52 trained models, each of them with discard of a link.

Each of the models trained with one Site discard and tried to predict the propagation models of the discarded Site. Some trained models correctly and others made mistakes, but a comparison has been made between sites measured and predicted by AI. Table IV shows the average error of the application of AI.

Table IV shows that the Coarse Tree application features an average error of $8.1 \mathrm{~dB}$, with $1.1 \mathrm{~dB}$ better than simply choosing the propagation model for the mean error, but the mean error of $8.1 \mathrm{~dB}$ is still very high.

Table III

Accuracy of each type of learning.

\begin{tabular}{|l|r|}
\hline \multicolumn{2}{|c|}{ Accuracy for each type of Classification Learner } \\
\hline Fine Tree & $\mathbf{3 8 . 5} \%$ \\
\hline Medium Tree & $\mathbf{3 8 . 5} \%$ \\
\hline Coarse Tree & $\mathbf{1 7 . 3 \%}$ \\
\hline Linear Discriminant & $\mathbf{1 0 0 . 0} \%$ \\
\hline Quadratic Discriminant & Falha \\
\hline Linear SVM & $\mathbf{6 3 . 5 \%}$ \\
\hline Quadratic SVM & $\mathbf{1 0 0 . 0} \%$ \\
\hline Cubic SVM & $\mathbf{1 0 0 . 0 \%}$ \\
\hline Fine Gaussian SVM & $\mathbf{1 0 0 . 0 \%}$ \\
\hline Medium Gaussian SVM & $\mathbf{9 8 . 1 \%}$ \\
\hline Coarse Gaussian SVM & $\mathbf{1 5 . 4 \%}$ \\
\hline Fine KNN & $\mathbf{1 0 0 . 0} \%$ \\
\hline Medium KNN & $\mathbf{1 5 . 4 \%}$ \\
\hline Coarse KNN & $\mathbf{7 . 7 \%}$ \\
\hline Cosine KNN & $\mathbf{1 5 . 4 \%}$ \\
\hline Cubic KNN & $\mathbf{1 5 . 4 \%}$ \\
\hline Weighted KNN & $\mathbf{1 0 0 . 0} \%$ \\
\hline Boosted Trees & $\mathbf{1 0 0 . 0 \%}$ \\
\hline Bagged Trees & $\mathbf{1 0 0 . 0 \%}$ \\
\hline Subspace Discriminant & $\mathbf{9 8 . 1 \%}$ \\
\hline Subspace KNN & $\mathbf{1 0 0 . 0 \%}$ \\
\hline RUS Boosted Trees & $\mathbf{6 5 . 4 \%}$ \\
\hline & \\
\hline & \\
\hline
\end{tabular}

Table IV

Average error of each Classification Learner.

\begin{tabular}{|l|r|}
\hline \multicolumn{2}{|c|}{ AI Average (dB) } \\
\hline Coarse Tree & $\mathbf{8 . 1}$ \\
\hline Weighted KNN & $\mathbf{8 . 3}$ \\
\hline Fine Gaussian SVM & $\mathbf{8 . 4}$ \\
\hline Cubic KNN & $\mathbf{8 . 7}$ \\
\hline Linear SVM & $\mathbf{8 . 8}$ \\
\hline Quadratic SVM & $\mathbf{9 . 0}$ \\
\hline Fine KNN & $\mathbf{9 . 2}$ \\
\hline Coarse KNN & $\mathbf{9 . 4}$ \\
\hline Bagged Trees & $\mathbf{9 . 6}$ \\
\hline Coarse Gaussian SVM & $\mathbf{9 . 6}$ \\
\hline Fine Tree & $\mathbf{9 . 8}$ \\
\hline Medium Tree & $\mathbf{9 . 8}$ \\
\hline Cosine KNN & $\mathbf{1 0 . 1}$ \\
\hline Cubic SVM & $\mathbf{1 0 . 3}$ \\
\hline Medium KNN & $\mathbf{1 0 . 4}$ \\
\hline Medium Gaussian SVM & $\mathbf{1 0 . 6}$ \\
\hline Boosted Trees & $\mathbf{1 1 . 1}$ \\
\hline Subspace KNN & $\mathbf{1 1 . 6}$ \\
\hline RUS Boosted Trees & $\mathbf{1 3 . 0}$ \\
\hline Subspace Discriminant & $\mathbf{1 3 . 3}$ \\
\hline Linear Discriminant & $\mathbf{1 4 . 8}$ \\
\hline
\end{tabular}

Some sites have very high errors. analyzing only the high errors of the best general average does not allow to conclude that this is a field problem or problem of the propagation model not suitable for the given situation.

When analyzing each case individually in the AI application, it was possible to observe that some Sites force errors of other Sites, without it being possible that the AI finds a relation that approximates the hit. It is concluded that these 
Sites have some problems, either of field or prediction of the model of propagation.

The tests were remade, with disposal of these Sites with problems. The Sites discarded were 03, 04, 05, 07, 10, 18 and 20, coming from Rio de Janeiro at Morro do Sumaré, 20 and 21 coming from São Gonçalo, 36 coming from Queimados, 37 coming from Nova Iguaçu, 40 coming from Pedra de Guaratiba and 22 e 23 coming from Rio Bonito. The number of Sites has been reduced to 38 .

With this new database, 38 trained models were created, discarding each of the links. The Accuracy for each type of learning can be seen in Table V.

Table V

Average error of each type of Classification Learner after correction of errors.

\begin{tabular}{|l|r|}
\hline \multicolumn{2}{|c|}{ AI Average (dB) } \\
\hline Weighted KNN & $\mathbf{4 . 6}$ \\
\hline Bagged Trees & $\mathbf{5 . 0}$ \\
\hline Fine KNN & $\mathbf{5 . 5}$ \\
\hline Linear SVM & $\mathbf{6 . 2}$ \\
\hline Boosted Trees & $\mathbf{6 . 4}$ \\
\hline Fine Gaussian SVM & $\mathbf{6 . 5}$ \\
\hline Coarse KNN & $\mathbf{7 . 0}$ \\
\hline Medium Gaussian SVM & $\mathbf{7 . 0}$ \\
\hline Coarse Tree & $\mathbf{7 . 1}$ \\
\hline Cubic KNN & $\mathbf{7 . 2}$ \\
\hline Medium KNN & $\mathbf{7 . 3}$ \\
\hline Subspace KNN & $\mathbf{7 . 7}$ \\
\hline Fine Tree & $\mathbf{7 . 8}$ \\
\hline Medium Tree & $\mathbf{7 . 8}$ \\
\hline RUS Boosted Trees & $\mathbf{8 . 1}$ \\
\hline Coarse Gaussian SVM & $\mathbf{8 . 1}$ \\
\hline Linear Discriminant & $\mathbf{8 . 1}$ \\
\hline Cosine KNN & $\mathbf{8 . 3}$ \\
\hline Quadratic SVM & $\mathbf{8 . 4}$ \\
\hline Cubic SVM & $\mathbf{8 . 9}$ \\
\hline Subspace Discriminant & $\mathbf{9 . 3}$ \\
\hline
\end{tabular}

In Table $\mathrm{V}$, it is verified that the Weighted $\mathrm{KNN}$ application presented a mean error of $4.6 \mathrm{~dB}$, with $4.6 \mathrm{~dB}$ better than simply choosing the propagation model by the mean error.

\section{CONCLUSION}

When comparing the field measurements of the Rio de Janeiro coverage area to the field prediction of the Progira software, it was possible to identify that the ITUR propagation model P.1812-3 in the geographic region option Dense Urban presents the smallest average error and is the one that best suits the characteristics of the relief.

When applying the IA tool, Classification Learner, it was possible to find a small improvement of the average error, of $1.1 \mathrm{~dB}$, however, when analyzing the Sites measured in isolation, some Sites force errors of other Sites, without it is possible that the AI finds a relation that approximates the hit. These sites have some problem, whether in the field measurement, transmission equipment or propagation model prediction.

By identifying these sites, treating them as problematic and suppressing them in the AI application, the accuracy shows a significant improvement with $4.6 \mathrm{~dB}$ rather than simply choosing the propagation model for the average error.

Weighted KNN is one of the Classification Learner approximation types that presented $100 \%$ accuracy in the network training and had the best performance in the comparison between the signal measured in the field and simulated in software, with a mean error of $4.6 \mathrm{~dB}$.

The low error indicates that the AI presents a good correlation, very close to the correct understanding of the characteristic of each point. This average error considerably increases the predictive capacity of the coverage area.

\section{REFERENCES}

[1] DONZELLI, V. Polarização Elíptica: Influência no Desempenho de Cobertura da TV Digital; 2011. 83 f. Dissertação (Mestrado em Engenharia Elétrica) Universidade Presbiteriana Mackenzie, São Paulo, 2011.

[2] LM TELECOM. 2018. Online. Available in: http://www.lmtelecom.com.br/, accessed 28 april 2018.

[3] MATSumoto, E. Y.; Matlab 6.5, Fundamentos de Programação: Editora_Erica.

[4] ABNT NBR 15604:2007. 2018. Online. Available in: http://www.abnt.org.br, accessed 28 april 2018.

[5] ORDINANCE N N $^{\circ} 925$, de 22 de agosto de 2014, publicado no D.O.U de 27 de agosto de 2014.

[6] ABNT NBR 15601:2007. 2018. Online. Available in: http://www.abnt.org.br, accessed 28 april 2018.

[7] LI W.; FELLOW Y. W.; ZHANG L.; LAFLÈCHE K. S. S.; HE D.; WANG Y.; GUAN Y.; ZHANG W.; MONTALBAN J.; ANGUEIRA P.; VELEZ M.; PARK S.; LEE J.; KIM H., Using LDM to Achieve Seamless Local Service Insertion and Local Program Coverage in SFN Environment, IEEE Transactions on Broadcasting, Vol. 63, $\mathrm{N}^{\mathrm{o}} 1$, march 2017.

[8] MATTSSON A.: Single Frequency Networks in DTV: IEEE Transactions on Broadcasting, Vol. 51, No 4, 2005.

[9] RODRIGUES C. F. F.; PERES F. de A.; LOVISOLO L.: Receivers Behavior in a ISDB-T's Single Frequency Network, SET International Journal of Broadcast Engineering - SET IJBE, V2, Article 7, 10p., 2016.

[10] ROSA, D. C. de O.; J_UNIOR, G. A. de D.: Reforçadores de sinais para melhoramento da cobertura do sinal de TV digital na região metropolitana da cidade de Goiânia, Revista de Radiodifusão, v. 08, n. 09, 2014.

[11] MARTINS R. A.: Modelagem e medições de onda de radio para predição de perda de propagação em ambientes urbanos: Tese (Doutorado em Engenharia Elétrica) Universidade Federal do Rio Grande do Norte, Natal, 2006.

[12] PROGIRA, SOME OF OUR CLIENTS. 2018. Online. Available in: www.progira.com/aboutus/some-of-ourclients/, accessed 28 april 2018. 
[13] ArcGis. Online. Available in: https://www.esri.com/enus/arcgis/about-arcgis/overview, accessed 28 april 2018.

[14] INTERNATIONAL TELECOMMUNICATION UNION, ITU-R P.1546-5: Recommendation ITU-R P.15465 , Method for point-to-area predictions for terrestrial services in the frequency range $30 \mathrm{MHz}$ to $3000 \mathrm{MHz}, 2013$.

[15] LUIZ A.V. P.; ASSIS M. S.: A Hybrid Prediction Model for Propagation Over Irregular Terrain in the VHF and UHF Bands, IEEE Latin America Transactions, VOL. 13, N 9, 2015.

[16] ANATEL: Anexo à Resolução número 284, de 7 de dezembro de 2001.

[17] MOSAICO. 2018. Online. Available in: http://sistemas.anatel.gov.br/se/public/view/b/srd.php, accessed 10 may 2018.

[18] SANTOS, F. R. D.: Comunidades Virtuais Baseadas em Vídeo Digital: Uma proposta de conteúdo adaptativo pautada em redes de aprendizagem e agentes inteligentes 2010.

[19] RUSSEL, S.; NORVIG P.: Inteligência Artificial, Editora Campus, 2013.

[20] REZENDE, S. O.: Sistemas Inteligentes, Editora Manole, 2013.

[21] BASHAR, A.; PARR G., MCCLEAN S.; SCOTNEY B.; Machine Learning based Call Admission Control Approaches: A Comparative Study: 2010 International Conference on Network and Service Management, 2010.

[22] SANTANA, I. R., PEREIRA, A. L. A Bioinspired Propagation Model for Brazilian Digital TV System based on MLP and RBF Networks, IEEE Latin America Transactions. Dissertação (Mestrado em Engenharia Elétrica) Universidade Presbiteriana Mackenzie, Volume: 14, Issue: 9 , setember 2016 .

[23] MALEKI, M.; MANSHOURI, N.; KAYIKC_IO_GLU, T.: Application of PLSR with a comparison of MATLAB classification learner app in using BCI, 2017, 25th Signal Processing and Communications Applications Conference (SIU), 2017.

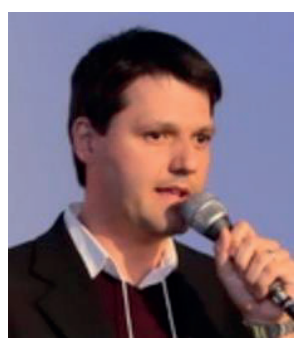

Alberto Leonardo Penteado Botelho holds a masters degree in electrical engineering from Mackenzie Presbyterian University, Has degree in Electrical Engineering from the Paulista University (UNIP), specialized in Engineering of Digital Television Systems by the National Institute of Telecommunications (INATEL), Engineering of Telecommunications Networks by the National Institute of Telecommunications (INATEL) and MBA in Project Management by Getúlio Vargas Foundation (FGV). Worked on Rede TV! and currently works at LM Telecom as a project engineer, where he has had the opportunity to work with Broadcast network projects since 2002 .

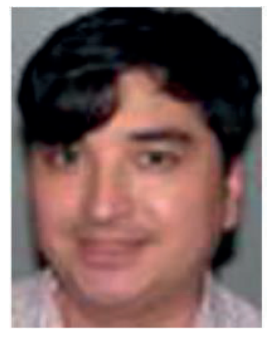

Cristiano Akamine received his B.Sc. degree in Electrical Engineering from Mackenzie Presbyterian University, Sao Paulo, Brazil, in 1999. He received his M.Sc. and Ph.D. degree in Electrical Engineering from the State University of Campinas (UNICAMP), Sao Paulo, Brazil, in 2004 and 2011 respectively. $\mathrm{He}$ is a Professor of Embedded Systems, Software Defined Radio and Advanced Communication at Mackenzie Presbyterian University. He is a Researcher in a Digital TV Research Laboratory at Mackenzie Presbyterian University since 1998, where he has had the opportunity to Work with many digital TV systems. His research interests are in a system on chip for broadcast TV and Software Defined Radio. 